\title{
Mental Training Enhances Attentional Stability: Neural and Behavioral Evidence
}

\author{
Antoine Lutz, ${ }^{1}$ Heleen A. Slagter, ${ }^{1,3}$ Nancy B. Rawlings, ${ }^{2}$ Andrew D. Francis, ${ }^{1}$ Lawrence L. Greischar, ${ }^{1}$ \\ and Richard J. Davidson ${ }^{1}$ \\ ${ }^{1}$ Waisman Laboratory for Brain Imaging and Behavior, University of Wisconsin-Madison, Madison, Wisconsin 53705, ${ }^{2}$ University Department of \\ Psychiatry, Warneford Hospital, Oxford OX3 7JX, United Kingdom, and ${ }^{3}$ Cognitive Neuroscience Group, Department of Psychology, University of \\ Amsterdam, 1018 WB Amsterdam, The Netherlands
}

The capacity to stabilize the content of attention over time varies among individuals, and its impairment is a hallmark of several mental illnesses. Impairments in sustained attention in patients with attention disorders have been associated with increased trial-to-trial variability in reaction time and event-related potential deficits during attention tasks. At present, it is unclear whether the ability to sustain attention and its underlying brain circuitry are transformable through training. Here, we show, with dichotic listening task performance and electroencephalography, that training attention, as cultivated by meditation, can improve the ability to sustain attention. Three months of intensive meditation training reduced variability in attentional processing of target tones, as indicated by both enhanced theta-band phase consistency of oscillatory neural responses over anterior brain areas and reduced reaction time variability. Furthermore, those individuals who showed the greatest increase in neural response consistency showed the largest decrease in behavioral response variability. Notably, we also observed reduced variability in neural processing, in particular in low-frequency bands, regardless of whether the deviant tone was attended or unattended. Focused attention meditation may thus affect both distracter and target processing, perhaps by enhancing entrainment of neuronal oscillations to sensory input rhythms, a mechanism important for controlling the content of attention. These novel findings highlight the mechanisms underlying focused attention meditation and support the notion that mental training can significantly affect attention and brain function.

\section{Introduction}

The capacity to sustain attention is critical for maintaining performance over a period of time. Although this capacity shows some heritability (Kuntsi and Stevenson, 2001), it is also influenced by factors such as fatigue and task familiarity (Aston-Jones et al., 1999). Deficits in sustained attention are a core feature of several mental illnesses, including attention-deficit hyperactivity disorder (ADHD) and schizophrenia (Winterer et al., 2000; Castellanos et al., 2006). Recent work has linked sustained attention impairment in these patient groups to increased trial-to-trial variability in reaction time and in neural responses (Winterer et al., 2000; Castellanos et al., 2006). In normal individuals, attention also frequently lapses, requiring a refocusing of attention. Such ongoing fluctuations in attention stability are thought to reflect competitive interactions between task-related and task-

Received April 3, 2009; revised Aug. 23, 2009; accepted Sept. 11, 2009.

This work was supported by grants from the National Institute of Mental Health (R.J.D.) and the National Center for Complementary and Alternative Medicine (R.J.D., A.L.) and gifts from Bryant Wangard and Ralph Robinson, Keith and Arlene Bronstein, and the John W. Kluge Foundation (R.J.D.). We thank the Barre Insight Meditation Society and P. Babe, S. Harkness, and Z. Moran for assistance in data collection and Matthieu Ricard, James Austin, and three anonymous reviewers for their comments on a previous version of this manuscript. We thank J. Davis for teaching the novices meditation. We also thank the Mind and Life Institute for helping to facilitate this work. A.L., H.A.S., and R.J.D. designed the study. A.L., H.A.S., N.B.R., and A.D.F. were involved in data collection. A.L., N.B.R., A.D.F., and L.L.G. analyzed the data. A.L. wrote the paper. All authors discussed the results and commented on the manuscript. Correspondence should be addressed to Antoine Lutz, Waisman Laboratory for Brain Imaging and Behavior, University of Wisconsin-Madison, 1500 Highland Avenue, Madison, WI 53705. E-mail: alutz@wisc.edu.

DOI:10.1523/JNEUROSCI.1614-09.2009

Copyright $\odot 2009$ Society for Neuroscience 0270-6474/09/2913418-10\$15.00/0 unrelated processes (e.g., mind-wandering) (Weissman et al., 2006; Kelly et al., 2008).

Contemplative traditions suggest that specific mental training regimens, such as a meditation practice that involves the focusing of attention [focused attention (FA) meditation], enhance the stability of attention and reduce the need to invoke executive skills that regulate the focus of attention from moment to moment (Gyatso et al., 1995; Gunaratana, 2002). Here, we investigated longitudinally whether 3 months of intensive meditation training, which included FA meditation as a major component, can increase attentional stability as one may predict based on traditional descriptions of FA. We probed attentional stability using a dichotic listening task that has been used previously to characterize attentional function in normal adults (Hillyard et al., 1973; Polich and Criado, 2006) and in patients with ADHD (Manassis et al., 1996) and schizophrenia (Winterer et al., 2000). Participants were presented with frequent standard and rare deviant tones in both ears and had to detect an intermittent deviant tone in the attended ear channel. We investigated the effects of FA meditation on moment-to-moment stability of attention by quantifying the cross-trial variability of (1) reaction time in response to attended deviant tones [SD of reaction time (RT)] (Castellanos et al., 2006)] and (2) the phase of oscillatory neural activity in response to attended and unattended deviant tones, as indexed by the phase-locking factor (PLF) (Palva et al., 2005). PLF is a frequency domain measure adapted specifically to measure trial-to-trial stability of stimulus-evoked neural responses. 
Several studies have shown that PLF is reduced in schizophrenic patients when compared with controls in similar auditory attention tasks, highlighting its potential relevance as a marker of attentional stability (Winterer et al., 2000).

Our main hypothesis was that intensive meditation training would significantly improve attentional stability. We predicted that intensive meditation training would reduce variability in attentional responses to deviant attended tones, as indicated by decreased SD of RT measurements and by increased PLF to target tones. In addition, we explored the possibility that FA meditation increases the monitoring of both target stimuli and distractions and thus affects stimulus processing in general. This unspecific meditation-related effect would be indicated by changes in PLF to attended and unattended deviant tones.

\section{Materials and Methods}

\section{Participants}

Seventeen practitioners (seven male; median age, 41 years; range, 22-64 years; median education, 18 years) were recruited before the start of a 3 month meditation retreat at the Insight Meditation Society (Barre, MA) (the practitioner group). Twenty-three matched controls (nine male; median age, 41 years; range, 20-62 years; median education, 17 years) with no previous meditation experience were recruited via advertisements in local newspapers directed at individuals interested in learning about meditation (the novice group). The mean age and the SD of age did not differ between groups (respectively, $t$ test, $t_{(1,29)}=-0.5$, $p=0.60$ and $\left.F_{(16,13)}=1.25, p=0.35\right)$. The participants had no history of mental or neurological illness and gave informed consent to participate. The practitioners self-selected for the meditation group and all had previous experience with meditation. Their average \pm SD life experience with meditation was $2967 \pm 3162 \mathrm{~h}$. This average includes daily practice and days in retreat (we counted $8 \mathrm{~h}$ of meditation per day of retreat). This research was approved by the University of Wisconsin-Madison institutional review board. Informed consent was obtained from all participants.

\section{Meditation training}

The practitioners participated in a 3 month meditation retreat during which they meditated for $10-12 \mathrm{~h} / \mathrm{d}$. Retreat participants were trained in Vipassana meditation [FA and open monitoring (OM) meditations (Lutz et al., 2008b)] and also received some training in "Metta," a loving kindness and compassion meditation. The novice group attended a $1 \mathrm{~h}$ group instruction class on Vipassana meditation before each visit to the laboratory and meditated daily for $20 \mathrm{~min}$. Their daily practice before data collection was assessed at each visit.

Vipassana meditation can be understood as a combination of two styles of practices that we conceptualized as FA meditation and OM meditation (Lutz et al., 2008b). The first style, FA meditation, entails voluntary focusing attention on a chosen object in a sustained manner. The second style, OM meditation, involves nonreactively monitoring the content of experience from moment to moment, primarily as a means to recognize the nature of emotional and cognitive patterns. The current study focused on the long-term impact of FA meditation. Our main assumption was that the mental processes trained during FA meditation are required for performance of sustained attention tasks, such as the dichotic listening task used in our study (for details, see below). FA meditation is a widespread style of Buddhist practice and involves sustaining selective attention moment by moment on a chosen object, such as a subset of localized sensations caused by respiration (for details, see Gunaratana, 2002). To sustain this focus, the practitioner must also constantly monitor the quality of attention. At the beginning of the practice, the attention may wander away from the chosen object, and the typical instruction is to recognize the wandering and then restore attention to the chosen object. For example, while intending to focus on localized sensations around the nostrils caused by breathing, one might notice that one's attention has been lost and that one has become unwillingly busy with thoughts unrelated to the task. One then simply releases this distraction and returns to the intended object. Thus, while cultivating the acuity and stability of sustained attention on a chosen object, this practice also develops three skills regulative of attention. The first is the monitoring faculty that remains vigilant to distractions without destabilizing the intended focus. The next skill is the ability to disengage from a distracting object without additional involvement. The last involves the ability to redirect focus promptly to the chosen object. Progress in this form of meditation is measured, in part, by the degree of effort required to sustain the intended focus. The novice contends with more distractions, and the three regulative skills are frequently exercised. As one advances, the three regulative skills can be developed to the point that, for example, advanced practitioners have an especially acute ability to notice when the mind has wandered. Eventually, FA induces a trait change, whereby the attention rests more readily and stably on the chosen focus. At the most advanced levels, the regulative skills are invoked less and less frequently, and the ability to sustain focus thus becomes progressively effortless.

\section{Stimuli and task}

Participants first performed an attention blink task to examine effects of intensive meditation on temporal attention (Slagter et al., 2007). This task was followed, after a 5 min break, by two versions of a standard selective attention auditory task, the dichotic listening task (Tiitinen et al., 1993). In one version ("focused attention version"), participants were instructed to attend to tone pips presented in one ear (e.g., the left ear) and to press a button each time they detected an intermittent deviant tone among the frequent ("standard") tones in that ear. They were asked to ignore concurrent tone pips in the opposite ear. Of importance, participants were explicitly instructed to do the focused attention task version as a FA meditation practice. In the second task version, participants were asked to press a button each time they detected a deviant tone in either ear [i.e., the left or the right ear; "open attention (OA) version"]. During this version of the task, they were asked to cultivate OM meditation (data not presented here). The order of the two task versions was randomized across participants. Participants were instructed to keep fixation on a central cross and to minimize eye movements and blinks during the experiment. They were sensitized to the issue of muscle artifacts by watching their electrophysiological (EEG) signal on the screen while moving their eyes and blinking and while squeezing their jaw muscles. Participants were asked to take this instruction as a recommendation rather than an additional task.

Each task version began with a 45 s eyes-open baseline, which was followed by a 3 min meditation [either FA meditation with the breath sensations as object (FA version) or OM meditation (OA version)]. This meditation period was followed by four 5-min blocks of 350 auditory stimuli ( $80 \mathrm{~dB}, 60 \mathrm{~ms}$ duration; rise and fall time, $10 \mathrm{~ms}$ each). The interstimulus interval was randomized between 700 and 1100 ms. Each block contained 300 standard (one ear, $1000 \mathrm{~Hz}$; the other ear, $500 \mathrm{~Hz}$ ) and 50 deviant (one ear, $1050 \mathrm{~Hz}$; the other, $475 \mathrm{~Hz}$ ) stimuli. The low and high tones were randomly presented in each ear across blocks in an order randomized across participants. Practitioners performed both versions of the dichotic listening task before and after the 3 month retreat. Novices also performed the two task versions twice with a 3 month period between sessions. The first session occurred 1 week after the first instruction class. The second session occurred $\sim 3$ months later, 1 week after the second instruction class.

\section{Electrophysiological data}

EEG was recorded at $512 \mathrm{~Hz}$ from $64 \mathrm{Ag}-\mathrm{AgCl}$ electrodes using the Active-Two system (BioSemi). With BioSemi systems, every electrode or combination of electrodes can be the "reference." We re-referenced the data offline to the average of both mastoids. Additional electrodes recorded the potential above the right and left mastoids and the electro-oculogram. The electro-oculogram was placed above and below the right eye.

\section{Data analyses}

Behavioral data analyses. We examined effects of intensive meditation training on several behavioral measures: the mean RT, the SD of RT, and the sensitivity index $\left(d^{\prime}\right)$, which indexes the ability to discern target stimuli (Swets et al., 1978). Fourteen practitioners and 17 novices were included in the behavioral and EEG analysis. Criteria of exclusions included misunderstanding of the instructions, data recording difficul- 
ties (battery or software failures, headphone malfunction), and participant dropout at time 2. Repeated-measures ANOVAs were run on RT, $\mathrm{SD}$, and $d^{\prime}$ with group (practitioners, novices) as a between-subject factor and time (time 1, time 2) as a within-subject factor. Because the SD might change depending on the mean RT, we also ran a control analysis on the SD normalized compared with the mean RT. Importantly, as when using the non-normalized SD values (see Results), we found a mental training-related reduction in reaction time variability as indexed by a significant group $\times$ time interaction when using the normalized SD values $\left(F_{(1,29)}=13.5, p<0.005\right)$. Trials in which a response was recorded to the standard tone immediately after a deviant tone that was faster relative to the onset of the standard tone than the fastest response measured to any deviant tone in the session were defined as "ambiguous" and not included in our analyses. Our behavioral results did not change when we included these ambiguous trials $(<0.08 \%$ on average across participants), as was shown in an additional control analysis (not reported here).

EEG data: analyses. EEGLAB (Delorme and Makeig, 2004) and Matlab (MathWorks) were used for offline EEG data processing. Data were bandpass filtered $(1-50 \mathrm{~Hz})$, re-referenced to the average of both mastoids, and cleared of large movement-related artifacts. ICA as implemented in EEGLAB (Delorme and Makeig, 2004) was then used to remove ocular and muscle artifacts [for a description of the method, see Delorme et al. (2007)]. Downsampled $(256 \mathrm{~Hz})$ data were epoched ( -1000 to $1500 \mathrm{~ms}$ after each tone) and baseline corrected (50 ms preceding tones). Trials with remaining artifacts (exceeding $\pm 100 \mu \mathrm{V}$ ) were removed. For each condition ("attended" and "unattended" deviant tones), group, and time point separately, the EEG signals were averaged in synchrony with tone onset to create event-related potentials (ERPs). Only trials used in the behavioral analysis were included in any EEG/ERP analysis.

\section{Phase-locking factor}

To investigate effects of FA meditation on the variability of brain responses, we examined stimulus locking of ongoing cortical activity using the PLF, which quantifies the non-uniformity of a phase distribution (Palva et al., 2005). PLF is an amplitude-independent measure and is, as such, less sensitive than the classical ERP measure to intertrial amplitude variability related to noise or small remaining artifacts, particularly for small number of trials, the reason being that each trial in the PLF approach is a unitary complex vector (i.e., amplitude equal to 1 ) and so contributes equally to mean PLF value. On the contrary, the amplitude of each trial used in the ERP analysis can fluctuate more as a result of a greater sensitivity to noise, small remaining artifacts, and small changes in impedance. PLF therefore provides a particularly sensitive measure of trial-to-trial stability of evoked responses. PLF ranges from 0 to 1 , with the value 1 indicating a delta-function distribution and the values approaching 0 indicating a uniform distribution. For $N$ complex values, $z i$, $i=1 \ldots N$, on the unit circle, the PLF is given by $N^{-1}\left|\sum z i\right|$ (Sinkkonen et al., 1995). The complex value $z i$ is computed as the complex phase of the Hilbert transform of a signal bandpass filtered in a frequency range of interest (Palva et al., 2005). If values $z i$ are uncorrelated and randomly drawn from a uniform distribution, the PLFs of different sets of $z i$ obey the Rayleigh distribution. The Rayleigh distribution is given by the following:

$$
p(\lambda)=\frac{\lambda}{\sigma^{2}} e^{-\frac{\lambda^{2}}{2 \sigma^{2}}}
$$

where

$$
\sigma=\frac{\lambda_{\mu}}{\sqrt{\pi / 2}},
$$

and $\lambda_{\mu}$ is the arithmetic mean of the distribution.

The null hypothesis that the samples were drawn from a uniform distribution was tested using the Rayleigh statistic in which $\lambda m$ is simply defined by the number of trials. Because the Rayleigh distribution is a function of its mean $(\lambda m)$ only, PLFs were normalized by $\lambda_{\mu}$ to express the strength of phase locking directly in a statistically meaningful manner (Palva et al., 2005). PLF/ $\lambda m>1.95$ corresponds to $p<0.05$ and $\mathrm{PLF} / \lambda m>2.42$ to $p<$ 0.01 . Normalized PLFs (nPLFs) were computed for the standard narrow-band frequency bands: delta $(1-3 \mathrm{~Hz})$, theta $(3-7 \mathrm{~Hz})$, alpha $(8-12 \mathrm{~Hz})$, beta $(13-30 \mathrm{~Hz})$, and gamma $(30-45 \mathrm{~Hz})$. Our first analysis focused on the average nPLF across frequency bands between 1 and $30 \mathrm{~Hz}$, because this frequency range contains most of the spectral power of the event-related potentials. In a second analysis, we examined mental training-related changes in phase locking for each of the different standard frequency bands separately. Gamma was not included in the broadband PLF analysis because we did not find any meditation training-related effects on the phase locking of the gamma frequency band.

Despite the normalization procedure, the PLF measure is sensitive to the number of trials used to compute phase locking. We therefore equated the number of trials used to compute PLF values between time (time 1, time 2) and condition (attention, unattended deviant tones) separately for each participant. To this end, we first determined the minimum number of artifact-free trials separately for each session and condition. The surplus number of trials was then randomly removed from the relevant conditions (e.g., attended deviant tones at time 1). In-house simulations showed that a minimum of 40 trials is necessary to obtain reliable phase-locking estimates (Slagter et al., 2009), and all participants had at least 40 trials of each condition at each session. The mean number of artifact-free trials used in the analysis was 79 for the practitioners and 78 for the novices. This number did not differ between the two groups $\left(t\right.$ test, $t_{(1,29)}=$ $-1.3, p>0.22$ ). To describe the meditation-related effect on PLF at each sample and channel, we submitted nPLF values to a repeated-measures ANOVA with time (time 1 or time 2) and condition (attended or unattended deviant tones) as withinsubject factors and group (practitioners or novices) as a betweensubjects factor (see Fig. $2 A, B$ ). The inference statistical analysis for the broadband analysis consisted of nine $t$ tests performed on the interaction term (group $\times$ time $\times$ condition) computed on the average PLF in three spatial regions (anterior, central, and posterior electrodes) and in three temporal intervals (50-150, 150-300, and 300-500). This selection was based on visual inspection of our data and on the latency and scalp topography of the classical ERP components N100, P200, and P300. We corrected for multiple corrections at $p<0.05$ using a nonparametric single threshold test (Nichols and Holmes, 2002). To this end, participants were randomly assigned 2000 times to two groups whose sizes were identical to the current group sizes. We computed two-tailed $t$ tests comparing the mean changes in PLF in attended versus unattended deviant tones between time 2 and time 1 for each of these regions and intervals $(3 \times 3$ tests $)$. We then computed the permutation distribution of the maximal voxel statistic over these nine tests. We rejected the omnibus hypothesis at level $\alpha=0.975$ if the maximal statistic for the actual labeling of the experiment is in the top $100{ }^{\star} \alpha \%$ of the permutation distribution for the maximal statistic. The thresholds used for correcting at $p<0.05$ were 3.1 for the group $\times$ time effect and 3.2 for the group $\times$ time $\times$ condition effect. The statistical analysis on the narrow-band analysis was similar but used $36 t$ tests (three spatial regions, three time intervals, and four frequency bands). The thresholds used for correcting at $p<0.05$ were 3.7 for the group $\times$ time effect and 3.8 for the group $\times$ time $\times$ condition effect. 


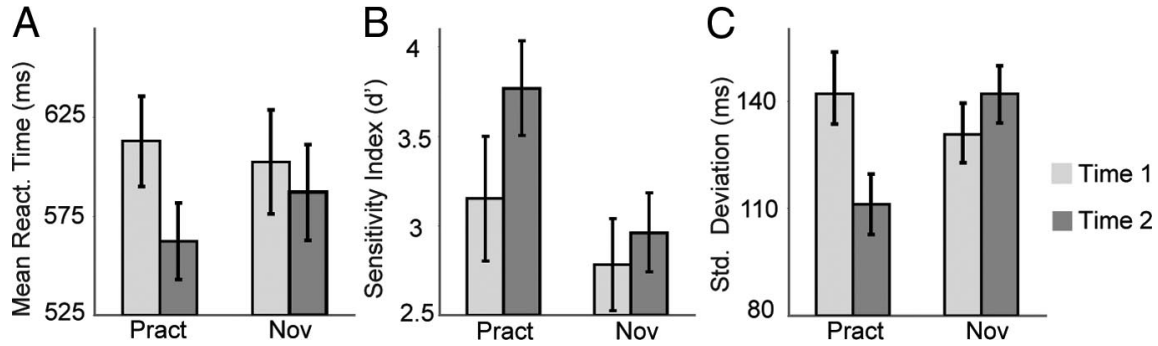

Figure 1. Intensive mental training reduces intra-individual variability of behavioral performance. Average mean reaction time $(\boldsymbol{A})$, sensitivity index $d^{\prime}(\boldsymbol{B})$, and SD of reaction time $(\boldsymbol{C})$ in response to target (attended deviant) tones, separately for each session (time 1, time 2) and group (practitioners, novices) ( $\pm \mathrm{SE}$ ). Note that the practitioners showed significantly lower variability of reaction times (SDs) than the novices at time 2 versus time 1.

\section{Event-related desynchronization}

The nonstimulus-locked amplitude fluctuations in oscillatory activity were obtained by time-frequency decompositions of EEG signals during the brain responses to deviant tones. Previous studies have shown most pronounced event-related desynchronization (ERD) in the alpha $(8-12$ $\mathrm{Hz}$ ) and beta $(13-30 \mathrm{~Hz}$ ) frequency bands (Pfurtscheller and Lopes da Silva, 1999). We therefore focused our ERD analysis on these bands. The amplitude envelope was computed by taking the amplitude of the Hilbert transform of the signal bandpass filtered in these frequency ranges. For each electrode and each frequency separately, we averaged the power of the amplitude envelopes across trials for each condition (attention vs unattended deviant tones) and each time (time 1 vs time 2). The average envelope was then convoluted to a unitary vector corresponding to a time window of $100 \mathrm{~ms}$ so as to smooth the envelope. ERD was then calculated by converting the power of the mean amplitude envelope at each frequency into percentage signal change relative to the average power in a reference prestimulus interval from -600 to $-200 \mathrm{~ms}$. We chose the baseline interval far from the onset of the stimulation (at $-200 \mathrm{~ms}$ ) to prevent the baseline from being affected by the induced response. To describe the meditation-related effect on ERD, we applied repeatedmeasures ANOVA with time (time 1 or time 2) and condition (attended or unattended deviant tones) as within-subject factors and group (practitioners or novices) as a between-subjects factor in alpha and beta frequency bands. The inference statistical analysis consisted of six $t$ tests performed on the interaction term (group $\times$ time $\times$ condition) computed from the average ERD in three spatial regions (anterior, central, and posterior electrodes) and in two temporal intervals (250-500 and $500-750 \mathrm{~ms})$. As above, we corrected these tests for multiple comparisons corrected at $p<0.05$. The threshold used for correcting at $p<0.05$ was 3.0 for the group $\times$ time $\times$ condition effect. These temporal intervals were chosen around the peak of the ERD, which occurred at $500 \mathrm{~ms}$ (see Fig. 5).

\section{Results}

We collected data from 17 participants at the beginning and end of a 3 month meditation retreat, during which participants meditated for $10-12 \mathrm{~h} / \mathrm{d}$ (practitioner group) (for details on the retreat, see Materials and Methods). We also collected control data from 23 participants interested in learning to meditate (novice group), who attended a $1 \mathrm{~h}$ meditation class and were asked to meditate for 20 min daily for 1 week before each session. Participants performed the dichotic listening task (see Materials and Methods) as a variation of FA meditation on breath sensations. Specifically, they were asked to sustain attention from moment to moment on the attended side to detect target tones, while also constantly monitoring the quality of this attention. We examined our predictions using separate repeated-measure ANOVAs with behavioral performance (RT, SD, and $d^{\prime}$ ), PLF, and ERD as the dependent measure and the between-subject variable group (experts vs novices) and within-subject variables time (time 1 or time 2) and/or condition (attended or unattended).

\section{Behavior}

We found that both groups performed better at the task at time 2 versus time 1 . These generic practice effects were reflected in faster mean reaction times (main effect of time, repeated-measures ANOVA, $\left.F_{(1,29)}=5.9, p<0.05\right)($ Fig. $1 A$ ) and increased target detection rates, as indexed by $d^{\prime}$ (main effect of time, $F_{(1,29)}=$ 7.3, $p<0.01$ ) (Fig. 1B). The overall increase in $d^{\prime}$ was produced by an increase in hit rates at time 2 (main effect of time, $\left.F_{(1,29)}=8.6, p<0.01\right)$. For experts, the mean \pm SD hit rates were $80 \pm 20 \%$ at time 1 and $91 \pm 10 \%$ at time 2 . For novices, the mean $\pm \mathrm{SD}$ hit rates were $81 \pm$ $18 \%$ at time 1 and $84 \pm 17 \%$ at time 2 . There was no significant change in false alarm rates over time in either group (paired $t$ test, $t_{(1,13)}=-1.7, p=0.1$ for practitioner group; $t_{(1,16)}=0.1, p=0.9$ for novice group). In addition, next to these generic practice effects, and in line with our prediction, we found that intensive mental training reduced behavioral response time variability. Thus, the practitioners showed a significantly greater reduction in RT variability (SDs) than the novices at time 2 versus time 1 (group $\times$ time interaction, repeated-measures ANOVA, $F_{(1,29)}=$ $10.2, p<0.005$ ) (Fig. $1 C$ ). This effect was driven by the practitioner group only (paired $t$ test on the difference in SD at time 2 versus time $1, t_{(1,13)}=3.1, p<0.01$; novices, $t_{(1,16)}=-1.3, p=$ 0.2 ). Mental training did not affect mean RT (group $\times$ time interaction effect, $F_{(1,29)}=1.8, p=0.18$ ) or target tone detection rates (group $\times$ time interaction effect, $F_{(1,29)}=1.97, p=0.17$ ). Of importance, there were no differences between groups in performance at time 1 (RT, SD, or $d^{\prime}$ measures: no main effect of group, all $p$ values $>0.28$ ).

\section{Phase-locking factor and event-related potentials}

We next predicted that the observed FA meditation-related reduction in reaction time variability would be associated with reduced variability of target-evoked brain responses, as indexed by the PLF (Palva et al., 2005). PLF is an amplitude-independent measure (i.e., PLF depends on the phase but not on the amplitude of the spectral representation of the signal; see Materials and Methods) adapted specifically to measure trial-to-trial stability of stimulus-evoked brain responses. To describe mental trainingrelated effects on neural response variability, we first ran a repeated-measures ANOVA for selected electrodes and time windows separately, using the average nPLF across frequency bands from 1 to $30 \mathrm{~Hz}$ as the dependent measure (see Materials and Methods). The analysis of stimulus locking of broadband phase allows the evaluation of the precise time course of the phaselocked events but does not disclose the spectral characteristics of the underlying activities. Therefore, we examined, in addition, stimulus locking within standard narrow-band frequency bands (delta, 1-3 Hz; theta, 4-7 Hz; alpha, 8-13 Hz; beta, 13-30 Hz). To correct for multiple comparisons, we used a nonparametric single threshold test (Nichols and Holmes, 2002). To reduce the number of tests, statistical examination of effects of FA meditation focused on three spatial regions (anterior, central, and posterior electrodes), three temporal intervals (50-150, 150-300, and $300-500 \mathrm{~ms}$ ), and four frequency bands. This selection was based on visual inspection of the data and the topography and chronomometry of standard evoked brain responses (N100, P200, and P300, respectively) (see Materials and Methods). 
To test our hypothesis that intensive FA meditation would enhance attentional stability at the neural level, we examined whether FA meditation affected the phase relationship between the recorded signals at a given latency and the presented stimuli across the trials, using the PLF. Mental training-related increases in phase locking of broadband $(1-30 \mathrm{~Hz})$ neural activity to attended deviant tones did not survive correction for multiple comparisons, yet narrow-band frequency analyses showed that, in line with our prediction, mental training was associated with decreased cross-trial variability in the phase of oscillatory activity in the theta band between 300 and $500 \mathrm{~ms}$ after attended deviant tones over frontal scalp regions, as indicated by a significant three-way interaction [group $\times$ time $\times$ condition (attended, unattended deviant); $F_{(1,29)}=5.7$, corrected $p<0.05$; see Materials and Methods]. This effect peaked $\sim 400 \mathrm{~ms}$ over frontal scalp regions (Fig. $2 A, B$ ), and was only found for the practitioner group (Fig. 2C) (paired $t$ test for practitioners on the difference time 2 vs time 1 attended versus unattended, between 300 and $500 \mathrm{~ms}$ over anterior electrodes, $t_{(1,13)}=3.1, p<0.01$; for the novices, $\left.t_{(1,16)}=-1.7, p=0.1\right)$. Importantly, and further in line with our hypothesis that mental training would reduce sustained attention task performance variability by enhancing the stability of cortical signal processing, these training-related changes in theta PLF predicted the observed training-related reduction in trial-to-trial reaction time variability (Fig. $2 D$ ). Specifically, between 300 and $500 \mathrm{~ms}$ (Fig. 2B), over anterior scalp regions, a negative cross-subject correlation was observed between the increase across sessions in the consistency with which the brain responded to attended versus unattended deviant tones and the decrease in RT variability to attended deviant tones across sessions (Fig. 2D) $(r=-0.40, p<0.05$ for practitioners and novices). Such a relationship was not seen with mean reaction time $(r=-0.14, p=0.46)$ or $d^{\prime}(r=0.13, p=0.45)$. To summarize, mental training reduced variability in attentional responses to task-relevant stimuli, as indicated by increased PLF to target tones and reduced reaction time variability.

In the dichotic listening paradigm, discrimination between target and nontarget stimuli usually produces an event-related potential in response to target stimuli (called the P3a) that is sensitive to demands of attention (Polich, 2007). The P3a is thought to reflect stimulus-driven disruption of frontal attention engagement (Polich, 2007). Notably, this component has a similar latency and scalp topography as the observed mental training-related increase in phase consistency to target tones, and we therefore explored effects of FA meditation on the amplitude of the P3a. In our data, replicating previous studies (for review, see Polich, 2007), we also observed a greater P3a to attended versus unattended deviant tones over frontal scalp regions between 300 and $500 \mathrm{~ms}$ (main effect of condition at electrode $\mathrm{Fz}$, $t_{(1,29)}=4.3, p<0.0002$, for all participants across sessions) (Fig. $3 A$ ). However, mental training did not affect P3a amplitude (group $\times$ time $\times$ condition interaction, $F_{(1,29)}=0.41, p=0.53$ at $\mathrm{Fz} ; F_{(1,29)}=2.1, p=0.16$ at $\mathrm{Cz}$; for practitioners, paired $t$ tests on $\mathrm{P} 3 \mathrm{a}$ amplitude comparing time 2 vs time 1 at $\mathrm{Cz}$ and $\mathrm{Fz}, p>0.5$ ). This is likely attributable to the fact that the ERP is more susceptible to trial-to-trial amplitude variability than PLF for relatively small numbers of trials and/or that this finding is specific to theta-band frequencies. In summary, mental training increased theta-band phase locking to target tones only, and this effect was not reflected in event-related potential measures of selective attention (P3a).

In addition, we explored the possibility that FA meditationgiven that it is also assumed to affect the processing of distractions
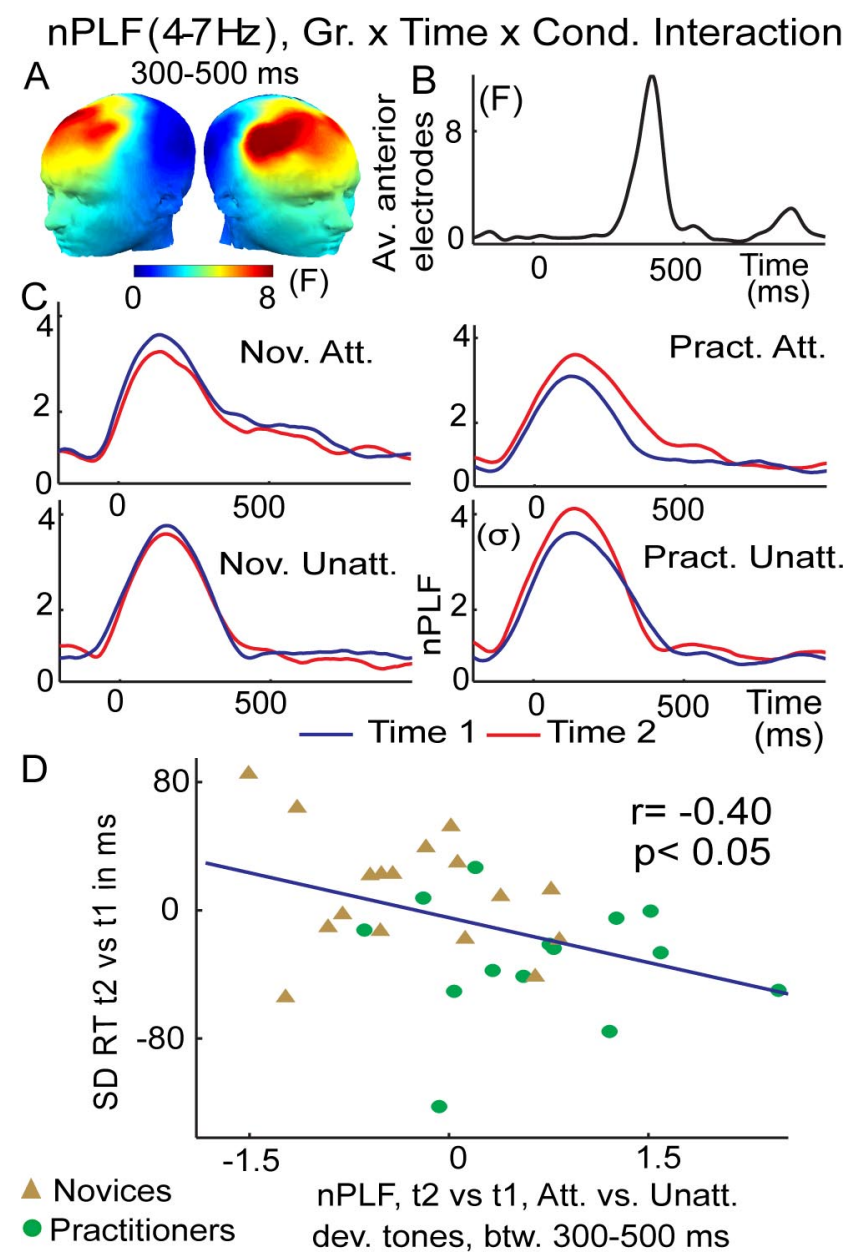

Figure 2. Intensive mental training increases trial-to-trial consistency of brain responses to attended deviant tones. $\boldsymbol{A}$, Spatial distribution of mental training-related increase in thetaband $(4-7 \mathrm{~Hz}$ ) phase consistency (indexed by normalized PLF) to target tones, as indexed by a three-way interaction between group (Gr.; practitioners, novices), time (time 1, time 2), and condition (Cond; attended, unattended deviant tones). Fvalues are averaged between 300 and 500 ms. $\boldsymbol{B}$, Time course of this three-way interaction across these significant electrodes. C, Normalized PLF values averaged across the electrodes sites showing a significant three-way interaction in $\boldsymbol{A}$ and $\boldsymbol{B}$, separately for the practitioners (Pract) and novices (Nov) and attention (Att) and unattended (Unatt) deviant tones. Note that the observed increase in phase consistency to attended deviant tones over time was only observed for the practitioner group. $\boldsymbol{D}$, The correlation plot shows that the observed change in the trial-to-trial variability of brain responses, as indexed by $\mathrm{nPLF}$, predicted the observed behavioral change in the trial-to-trial variability of the RT (SD).

and task-unrelated thought-may affect stimulus processing in general (see Materials and Methods) (Lutz et al., 2008b). Specifically, we examined whether FA meditation may modulate both distracter and target processing, as indicated by changes in PLF to deviant tones regardless of whether they were presented in the attended or unattended ear channel. Indeed, we found a robust meditation-related increase in broadband phase locking of neural activity at frontal electrodes between 150 and $300 \mathrm{~ms}$ to any deviant tone (group $\times$ time interaction; on average $F_{(1,29)}=8.4$, $p<0.01$, corrected for multiple comparisons at $p<0.05$ ) (Fig. $4 A-C)$. The effect was driven by stronger phase locking to deviant tones at time 2 in the practitioner group (paired $t$ test on the different time 2 vs time 1 for unattended deviant tones, $t_{(1,13)}=$ 2.4, $p<0.05$ and attended deviant tones, $t_{(1,13)}=5.8, p<$ $0.0001)$, an effect that was not observed for the novice group $\left(t_{(1,16)}=-1.1, p=0.27\right.$ and $t_{(1,16)}=-0.42, p=0.68$, respec- 


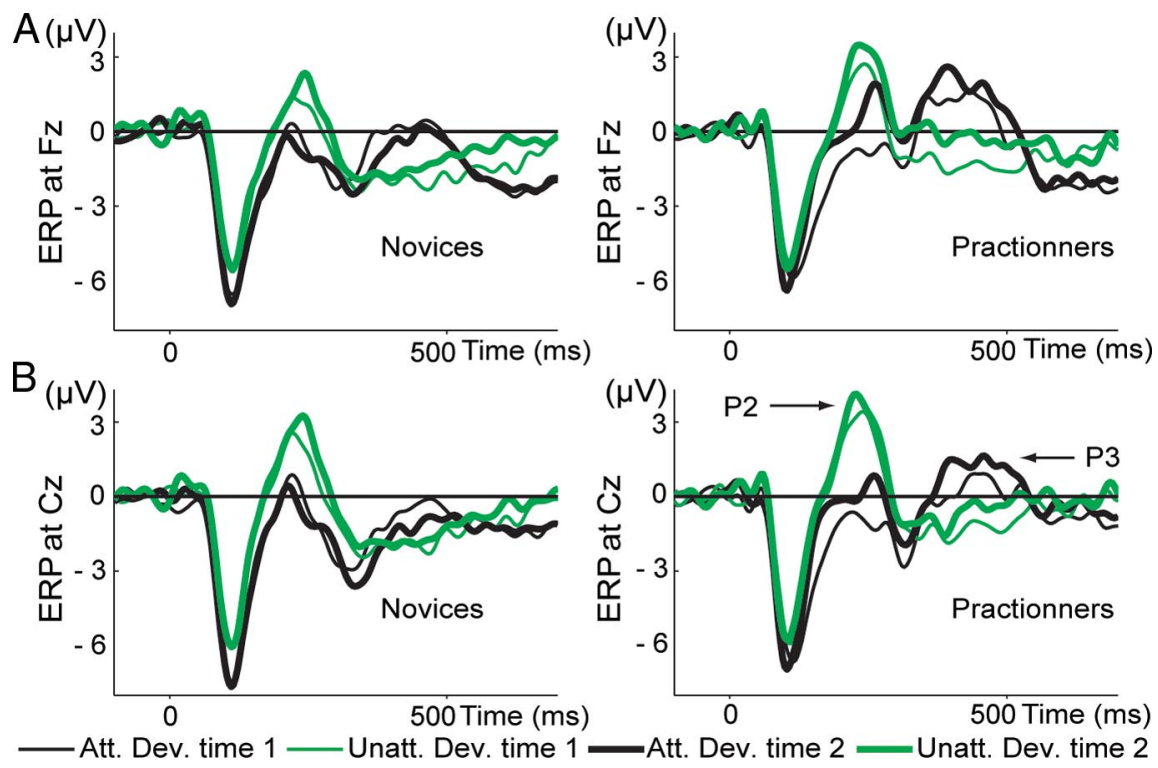

Figure 3. ERPs. Shown are ERPs $(1-30 \mathrm{~Hz})$ to attended (black line) and unattended (green line) deviant tones at $\mathrm{Fz}(\boldsymbol{A})$ and $\mathrm{Cz}$ $(\boldsymbol{B})$ at time 1 (thin line) and time 2 (thick line), separately for the novices (left) and the practitioners (right). In line with previous studies, a smaller P200 (averaged between 150 and $300 \mathrm{~ms}$ ) was observed to attended (Att.) versus unattended (Unatt.) deviant (Dev.) tones (main effect of condition, $t_{(1,30)}>6.4, p<1.10^{-6}$ above $\mathrm{Fz}$ and $\mathrm{Cz}$ ). The amplitude of the P200 increased across conditions only for the practitioners at time $2\left(t_{(1,13)}>2.7, p<0.05\right.$ over $\mathrm{Fz}$ and $\left.\mathrm{Cz}\right)$. Note that, in line with previous studies, attended target tones elicited a bigger P3a than unattended tones (main effect of condition over electrode $\mathrm{Fz}, t_{(1,29)}=4.3, p<$ 0.0002 , for all participants across sessions).

tively). The observed meditation-related increase in broadband phase-locking PLF effects to attended deviant tones also predicted corresponding changes in intra-individual variability in reaction time $(r=-0.50, p<0.005)$ (Fig. $4 D), d^{\prime}(r=0.48, p<$ $0.01)$, and reaction time $(r=-0.35, p=0.06)$. This early mental training-related PLF effect remained significant over anterior electrodes between 300 and $500 \mathrm{~ms}$, although the effect was weaker (corrected $p<0.05, F_{(1,29)}=5.9, p<0.05$ ).

Notably, the scalp topography and latency of the early mental training-related, attention-independent PLF effect corresponds to the scalp topography and latency of the P200 (Fig. 3B), as confirmed by an additional ERP analysis: the topography of the P200 (averaged between 150 and $300 \mathrm{~ms}$ ) positively correlated with the topography of the broadband PLF between 150 and 300 $\mathrm{ms}(r=0.48, p<0.0001$ for practitioners and $r=0.26, p<0.05$ for controls). For the practitioners only, the topography of the P200 variations between time 1 and time 2 correlated with the topography of the PLF variations between time 1 and time $2(r=$ $0.72, p<0.0001)$. The amplitude of the P200 increased for the practitioners at time 2 (paired $t$ test on time 2 vs time 1 at Fz, $t_{(1,13)}=2.8$, $p<0.05$ and at $\left.\mathrm{Cz}, t_{(1,13)}=2.7, p<0.05\right)$ but not for novices (paired $t$ test, time 2 vs time 1 for novices, at Fz, $t_{(1,16)}=0.7, p=$ 0.49 and at $\left.\mathrm{Cz}, t_{(1,13)}=0.5, p=0.66\right)$. These results suggest that mental training also may have affected processes reflected by the P200. The P200 is typically associated with the beginning of an executive process responsible for stimulus identification and the initiation of decision making and is also modulated by attention (Lindholm and Koriath, 1985). In line with previous studies, a smaller P200 (averaged between 150 and $300 \mathrm{~ms}$ ) was observed to attended versus unattended deviant tones (paired $t$ test for all participants attended versus unattended deviant tones averaged across time 1 and time $2, p<0.000001, t_{(1,30)}>6.4$ above $\mathrm{Fz}$ and $\mathrm{Cz}$ ). Because the PLF frontal effect remained significant during the interval 300-500 ms, we also explored possible mental training-related changes in ERPs in this later interval at electrodes
$\mathrm{Fz}$ and $\mathrm{Cz}$. Indeed, we found an increase in P3 amplitude for the practitioners only at time 2 (repeated-measures ANOVAs, group $\times$ time interaction on average ERP between 300 and $500 \mathrm{~ms}, F_{(1,29)}=4.5, p<$ 0.05 at $\mathrm{Fz}$ and $F_{(1,29)}=5.4, p<0.05$ at $\mathrm{Cz}$; paired $t$ test for time 2 vs time 1 for all deviant tones for practitioners, $t_{(1,13)}=2.3$, $p<0.05$ at $\mathrm{Fz}$ and $t_{(1,13)}=2.1, p=0.06$ at $\mathrm{Cz}$ and for novices, $t_{(1,16)}=-1.0, p=0.33$ at $\mathrm{Fz}$ and $t_{(1,16)}=-0.15, p=0.88$ at $\left.\mathrm{Cz}\right)$ (Fig. 3).

Finally, we also examined mental training-related changes in stimulus locking to any deviant tone within standard narrow-band frequency bands for the same electrode sites and time windows for which broadband effects were observed to shed light on the spectral characteristics of this effect. Mental training only affected phase locking to any deviant tone in the delta $(1-3 \mathrm{~Hz})$ frequency band (frontal electrodes, interval of 300-500 ms, $F_{(1,29)}$ $=8.2, p<0.05$ corrected). Notably, recent work suggests that, when taskrelevant input is regular or "rhythmic," attention can enforce oscillatory entrainment to the input stream, thereby optimizing performance (Jones and Boltz, 1989; Schroeder and Lakatos, 2009). As in our task, the stimulus presentation rate fell within the frequency of the delta band [with a mean stimulus onset asynchrony (SOA) of $950 \mathrm{~ms}$ ], an intriguing possibility is thus that the observed mental training-related modulation of general stimulus processing in the delta band reflects an increase in oscillatory entrainment to the task-input rhythmic of the auditory tones. To explore this possibility, we analyzed mental training-related effects of phase locking to any deviant tone separately for a frequency band centered on the task-input rhythmic (estimated to be $0.85-1.3 \mathrm{~Hz}$ based on the range of SOAs) and the higher part of the delta band $(1.5-3 \mathrm{~Hz})$ (for this analysis, the EEG signals were bandpass filtered between 0.5 and $30 \mathrm{~Hz}$ ). Both analyses replicated the above findings for the $1-3 \mathrm{~Hz}$ band, with mental training enhancing phase locking of oscillatory activity above frontal electrodes in both delta sub-bands $(0.85-1.3 \mathrm{~Hz}$ band, $t$ test comparing practitioners vs novices at time 2 vs time 1 , $\left.t_{(1,29)}=3, p<0.01\right)$, albeit more strongly so in the higher delta band $(1.5-3 \mathrm{~Hz})$ than in the task-input rhythmic band ( $t$ test, $\left.t_{(1,29)}=2.4, p<0.05\right)$. This effect was driven by the practitioners showing higher PLF amplitude in high delta band than $0.85-1.3$ $\mathrm{Hz}$ at time 2 compared with time 1 (paired $t$ test, $t_{(1,13)}=5, p<$ 0.005). Nevertheless, the fact that mental training enhanced phase locking of oscillatory activity in a band corresponding to the auditory input frequency suggests that FA meditation may have lead attention to operate more steadily in a rhythmic mode of attention.

One could also argue that observed increase in phase locking to unattended deviant tones simply reflects an increase in general arousal level for meditators at time 2 rather than an increase in monitoring of any deviant tone or a reduction in task-unrelated processes. Higher arousal is generally thought to increase the sensitivity of the system to any sensory stimulus and has typically been associated with a reduction in EEG power in the alpha band $(8-12 \mathrm{~Hz})$ (Niedermeyer, 1999). To 
exclude the possibility that our effects merely reflect differences between groups in changes in arousal over time, we computed the global EEG power in the alpha band. Importantly, we did not find a group $\times$ time effect (repeatedmeasures ANOVA, $\left.F_{(1,28)}=0.02, p=0.88\right)$. Rather, for both groups, we observed an increase in global alpha power (main effect of time, $F_{(1,28)}=4.6, p<0.05$; mean power in a $2 \mathrm{~s}$ window for practitioners, $2.9 \times 10^{-5} \mu \mathrm{V}^{-2}$ at time 1 and $4.1 \times 10^{-5} \mu \mathrm{V}^{-2}$ at time 2 , and for controls, $2.4 \times 10^{-5} \mu \mathrm{V}^{-2}$ at time 1 and $3.3 \times 10^{-5} \mu \mathrm{V}^{-2}$ at time 2). This finding argues against the possibility that a selective increase in arousal at time 2 in the practitioner group can explain our findings.

To summarize the above findings, mental training enhanced trial-to-trial phase consistency of oscillatory brain responses to attended deviant tones, as well as to deviant tones in general (i.e., independent of selective attention). The mental training-related increase in phase consistency to any deviant tone correlated with a mental training-related increase in the amplitude of the P200 ERP. These meditation-related effects could not simply be explained by a mere change in arousal level at time 2 for the meditators, because global alpha power increased similarly for both groups.

\section{Event-related desynchronization}

In addition, because we found previously that meditation training affects the distribution of limited brain resources (Slagter et al., 2007), we estimated how FA meditation affects cortical processing during the task. We indexed the extent of cortical engagement required to meet tasks demands by quantifying ERD to target tones. ERD reflects the blocking of alpha $(8-12 \mathrm{~Hz})$ and beta $(13-30 \mathrm{~Hz})$ EEG oscillatory rhythms by stimulus processing, in particular during motor preparation and execution, and is thought to reflect increased cellular excitability in thalamocortical systems during cortical information processing (Pfurtscheller and Lopes da Silva, 1999). Effort exerted during task performance, task difficulty, advanced age, and low intelligence quotient are all factors that enhance ERD (for review, see Pfurtscheller and Lopes da Silva, 1999). In contrast, cognitive task practice has been found to reduce beta ERD (Romero et al., 2008). In line with the hypothesis that FA practice results in a decreased need for active engagement (Lutz et al., 2008b), we found that intensive mental training was associated with a reduction in ERD of beta oscillatory activity to attended deviant tones over the three spatial regions of interest as reflected by a group $\times$ time $\times$ condition interaction between 500 and $750 \mathrm{~ms}\left(F_{(1,29)}=4.8, p<0.05\right.$; $F_{(1,29)}=5.2, p<0.05 ; F_{(1,29)}=4.7, p<0.05$, across anterior, middle, and posterior electrodes, respectively, $p<0.05$ corrected) (Fig. 5). This interaction was driven by the practitioners showing less ERD at time 2 in the attended condition only ( $t$ test comparing ERD to target minus ERD to unattended deviant tones at time 2 vs time $\left.1, t_{(1,13)}=3.4, p<0.005\right)$, although no such effect was observed for the novices $\left(t_{(1,16)}=-1.1, p=0.29\right)$ (Fig. 5). The peak of this effect was located over frontal and left centroposterior electrodes (Fig. 5A). The lateralization of this ERD pattern above somatosensory electrodes contralateral to the hand executing the motor response (paired $t$ test between the average across electrodes $\mathrm{CP} 1, \mathrm{CP} 3, \mathrm{CP} 5, \mathrm{P} 1, \mathrm{P} 3$, and $\mathrm{P} 5$ and the average across $\mathrm{CP} 2, \mathrm{CP} 4, \mathrm{CP} 6, \mathrm{P} 2, \mathrm{P} 4$, and $\mathrm{P} 6, t_{(1,30)}=-2.84, p<0.01$ ) is in line with the proposal that beta-band ERD in part reflects motor selection processes (Doyle et al., 2005). Different from the observed mental training-related increase in phase consistency, these mental training-related changes in ERD did not correlate with the observed mental training-related behavioral changes in
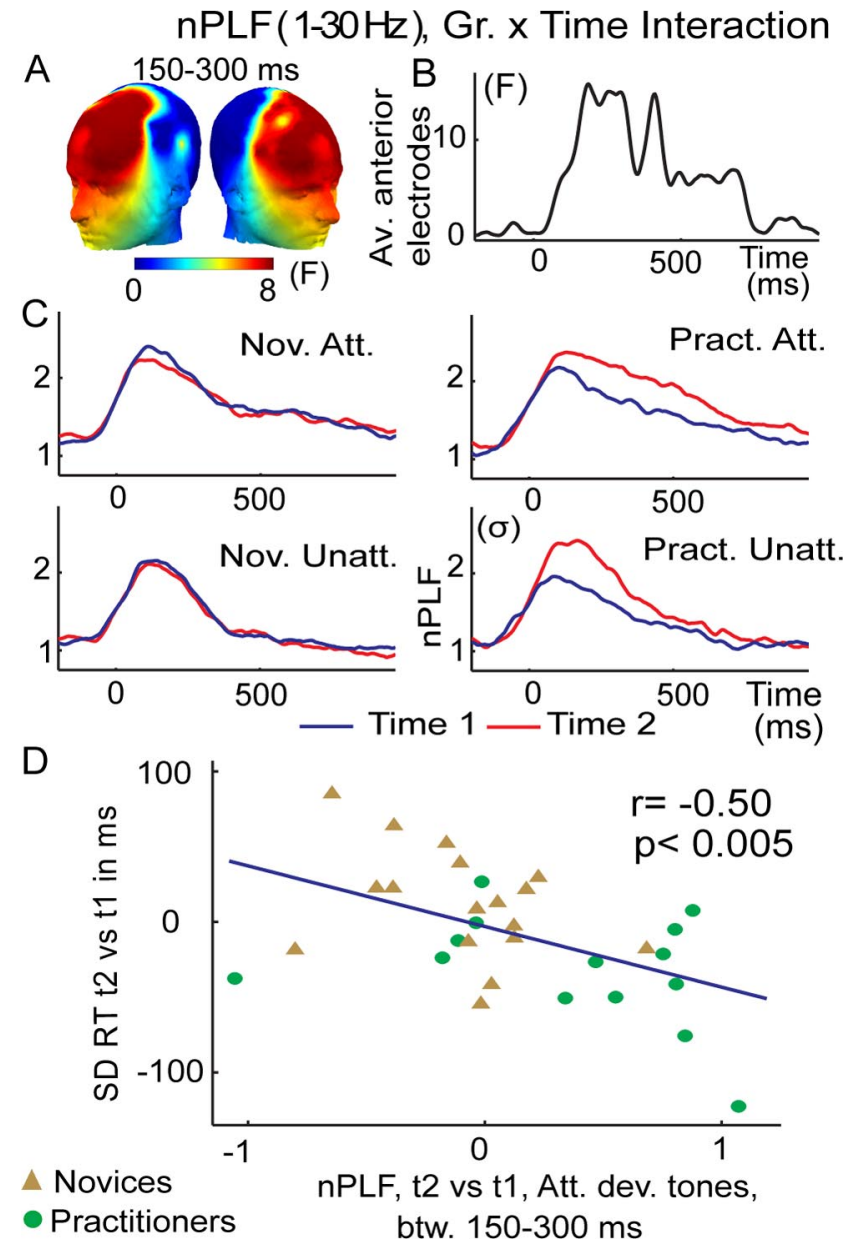

Figure 4. Intensive mental training increases trial-to-trial consistency of brain responses to any deviant tones. $\boldsymbol{A}-\boldsymbol{D}$, The graphs show the group $\times$ time interaction on broadband (1-30 $\mathrm{Hz}$ ) nPLF following the display conventions and analysis from Figure 2. A mental trainingrelated increase in phase locking to deviant tones in general (attended and unattended) was significant ( $p<0.05$, corrected) between 150 and 300 ms across the a priori group of frontal electrodes $(\boldsymbol{A}-\boldsymbol{C})$ and correlated negatively, for the attended deviant tones, with the observed reduction in RT SD $(\boldsymbol{D})$. A similar negative correlation $(r=-0.30)$ was found for the unattended deviant tones, even if this effect did not reach significance ( $p=0.11$ ).

attentional stability (i.e., variability of reaction time). This latter finding may indicate that the observed effects of mental training on the stability of attention and task engagement were independent. This may not be surprising given that ERD is thought to index the amount of cortical engagement allocated by the system to perform the task, rather than reflect the actual performance of the system during the task (Pfurtscheller and Lopes da Silva, 1999). Alpha ERD was not affected by mental training, although this frequency band showed the greatest reduction in ERD to attended versus unattended deviant tones in general (data not shown). To summarize, the observed mental training-induced changes in ERD of beta-band activity suggests a reduction in the amount of resources or engagement necessary to perform the task as a result of training.

\section{Discussion}

Our study examined whether 3 months of intensive mental training, as cultivated by FA meditation, can enhance (1) attentional stability as reflected by decreased trial-to-trial variability in brain and behavioral responses to deviant attended tones and (2) reduce resource allocation or cortical engagement (i.e., promote 


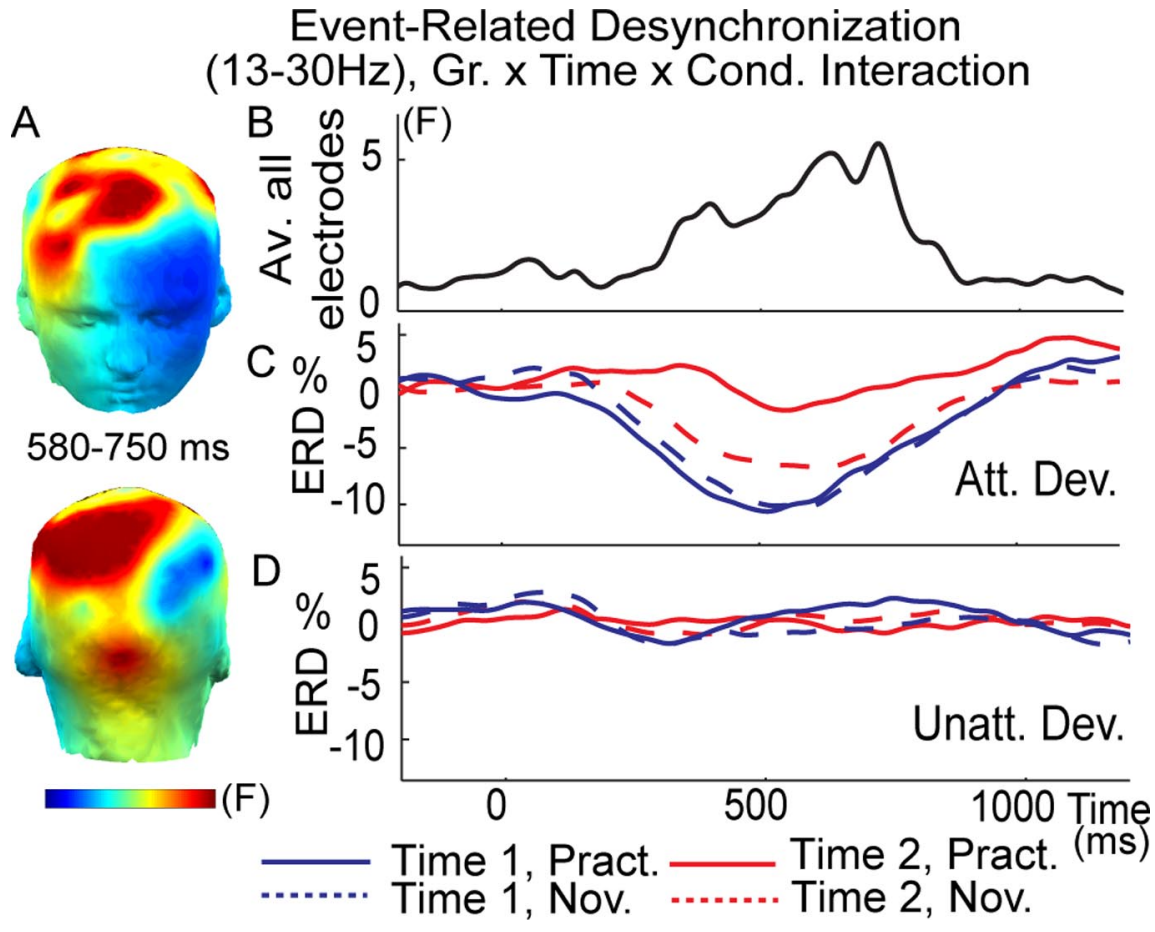

Figure 5. Mental training selectively reduced cognitive effort as indexed by ERD. $A$, Spatial distribution of the mental trainingrelated decrease in beta-band (13-30 Hz) ERD to attended (Att.) versus unattended (Unatt.) deviant (Dev.) tones [3-way interaction between group (Gr.; practitioners, novices), time (time 1, time 2), and condition (Cond; attended, unattended deviant tones)]. $F$ values are averaged between 500 and $750 \mathrm{~ms}$. Note that this interaction is significant $(p<0.05$, corrected), above the three a priori groups of anterior, central, and posterior electrodes. Time course of this three-way interaction ( $F$ value) $(\boldsymbol{B})$ and of the ERD for attended $(\boldsymbol{C})$ and unattended $(\boldsymbol{D})$ deviant tones (Dev.) on average across the three a priori groups of electrodes (anterior, central, and posterior) for the practitioners (Pract) and novices (Nov).

more efficient processing). There were three main findings. First, we found a mental training-related increase in phase consistency of theta-band oscillatory neural responses over anterior scalp regions to target stimuli only (Fig. 2). Importantly, this change in cortical signal stability predicted the observed reduction in reaction time variability (Fig. 1). Second, mental training reduced cortical engagement, as reflected by a reduction in ERD to target tones in the beta $(13-30 \mathrm{~Hz})$ frequency band (Fig. 5). Third and last, mental training was associated with enhanced phase consistency of the brain responses to any deviant tone (Fig. 4). Together, these novel findings confirm first-person reports that FA meditation can affect the stability of attentional processes and reveal the neural mechanisms underlying an improved ability to sustain attention. They extend previous work showing effects of a variety of meditation styles on various mental skills (BrefczynskiLewis et al., 2007; Jha et al., 2007; Slagter et al., 2007, 2009; Tang et al., 2007; Lutz et al., 2008a,b; Cahn and Polich, 2009) and corroborate the idea that purely mental training, as cultivated by meditation, can significantly affect brain and mental function (Lutz et al., 2008b). Below we will discuss these three principal findings and their implications in more detail.

\section{Effect of mental training on attentional stability}

In support of our main hypothesis, we found a mental trainingrelated increase in phase consistency of theta-band oscillatory neural responses over anterior scalp regions to target stimuli only, which predicted the observed reduction in reaction time variability. The observation that increases in theta phase locking over anterior regions were accompanied by less variable performance is consistent with a role of theta in cognitive control
(Hanslmayr et al., 2008) or in the formation of mnemonic neuronal representations (Jensen and Lisman, 1996; Kahana et al., 2001), important for sustaining attention on the task from moment to moment. Thus, the observed mental training-related increase in the consistency with which the brain can respond to a target stimulus from moment to moment may reflect increased cognitive control capacity, for example, an increased ability to suppress task-unrelated thoughts or counter distractions, in line with phenomenological descriptions of this technique (Lutz et al., 2008b). This would be reflected in increased cortical signal-tonoise (e.g., PLF) in networks important for sustaining a performance over time. Similar proposals have been made to characterize attention disorders in several major psychiatric disorders (Rolls et al., 2008). For instance, simulations using biologically realistic neural networks have related the EEG-measured reduction of cortical phase consistency in schizophrenia (Winterer et al., 2000) to a diminished stability of these networks and associated reduced network stability to alterations of dopamine levels in the prefrontal cortex (Loh et al., 2007). In addition to dopamine, noradrenergic projections originating from the locus ceruleus are also important for sensory signal transmission in cortical areas (Aston-Jones et al., 1999). Thus, the observed increase in phase consistency after intensive FA meditation training might be linked to cortical signal-to-noise of attentional networks in the brain and prefrontal dopaminergic and/or noradrenergic function. Future studies will need to incorporate brain measures of neurotransmitter functions (e.g., positron emission tomography) and first-person reports of mindwandering (Christoff et al., 2009) to explicitly test this assumption. To summarize, FA meditation may have improved signalto-noise ratios in neural networks involved in attention.

\section{Effect of mental training on cortical engagement}

In addition, we found that intense mental training affected cortical engagement, as reflected by a concomitant reduction in ERD to target tones in the beta $(13-30 \mathrm{~Hz})$ frequency band (Fig. 5). ERD is usually viewed as a correlate of increased cellular excitability in thalamocortical systems during cortical information processing (Pfurtscheller and Lopes da Silva, 1999). Within this framework, previous studies have interpreted reductions in beta ERD after practice of external tasks as decreased cognitive effort (Pfurtscheller and Lopes da Silva, 1999; Romero et al., 2008). A mental training-related reduction in task effort would be consistent with traditional accounts of progress in this practice (Gyatso et al., 1995; Gunaratana, 2002). At a more advanced level of training, the regulative attentional skills allegedly are invoked less and less frequently, and the ability to sustain focus thus becomes progressively "effortless" (for a recent discussion, see Lutz et al., 2008b). Previous functional magnetic resonance imaging findings from our group, which showed less blood oxygenation leveldependent activity in attention-related networks in expert 
meditators with the most hours of practice during FA meditation, also indicate that one long-term effect of FA meditation may be reduction in task effort (Brefczynski-Lewis et al., 2007). In the present study, this interpretation is limited, however, by the lack of first-person reports on perceived effort or of psychophysiological measures of cognitive effort (e.g., pupillometry). A more parsimonious reading of the finding is that mental training affected the oscillatory mode of the brain such that less cortical processing was needed to meet task demands. As discussed below, such change in neuronal oscillations of the system might be have been achieved by reducing task-irrelevant processes and/or by simply facilitating a sustained oscillatory mode throughout the task.

\section{Enhanced processing of task-related auditory inputs during FA meditation}

As mentioned above, in addition to increases in attention-related cortical signal stability, we also observed increases in stimulusrelated neural processing regardless of whether the deviant tone was attended or unattended. Focused attention meditation may thus affect both distracter and target processing. This possibility is consistent with phenomenological descriptions of this style of meditation. While cultivating the acuity and stability of sustained attention on a chosen object, this practice is also said to develop three skills regulative of attention (Gyatso et al., 1995; Gunaratana, 2002; Lutz et al., 2008b) (see Materials and Methods). The first is the monitoring faculty that remains vigilant to distractions without destabilizing the intended focus. The next skill is the ability to disengage from a distracting object without additional involvement. The last involves the ability to redirect focus promptly to the chosen object (Lutz et al., 2008b). FA meditation training is thus thought to result not only in a high frequency of moments of attention on the attended object (or increased attentional stability) but also to improve one's ability to remain vigilant and monitor distractors without losing focus. Consistently with this description of FA meditation effects, we observed a reduction in phase variability to any deviant tone during a time interval [150$300 \mathrm{~ms}$, i.e., before the selective mental training effect on attentional processing (300-500 ms)]. This general increase in phase consistency was focused over anterior scalp regions and accounted for $22 \%$ of the variance in behavior $(30 \%$ for the attended deviant tone) (Fig. 4). This effect was also highly similar in both time course and scalp topography to the P200 (Fig. 3). This ERP component is typically associated with the beginning of executive processes responsible for stimulus identification and the initiation of decision-making and is also modulated by attention (Lindholm and Koriath, 1985). This point is important if one assumes that monitoring of distractors is one important mechanism for regulating task-unrelated thoughts processes during task performance (Lutz et al., 2008b). The persistence of this effect in the later time interval (300-500 ms) for both PLF and ERP (P3) (Fig. 3) is consistent with this interpretation [for an opposite effect of meditation on the P3 to distractors, see Cahn and Polich (2009)].

As discussed above, it is possible that FA meditation reduced cortical signal-to-noise (e.g., PLF) in attention-related networks and that this stabilized responding to target and distracter stimuli alike. Another, albeit not mutually exclusive, possibility is that FA training led attention to operate more steadily into a rhythmic mode (Lakatos et al., 2008; Schroeder and Lakatos, 2009), in tune with the temporal structure of the stimuli. Temporal expectations of rhythmic sensory stimuli stream spontaneously form and can optimize task demands (Jones and Boltz, 1989). Typically, perceptual judgments are enhanced for stimuli occurring in trials with more regular rhythm (Jones et al., 2006; Schroeder and Lakatos, 2009). In our task, the auditory tones were presented with some temporal regularity, with the average time interval between the onset of a first stimulus and the onset of a second stimulus being $\sim 900 \pm 150 \mathrm{~ms}$. Thus, training to sustain attention could have led practitioners to better anticipate the temporal aspects of the auditory stream. Importantly, during such dynamic attending (Jones and Boltz, 1989) or rhythmic mode of attention (Schroeder and Lakatos, 2009), neural oscillations, in particularly low-frequency ones [e.g., delta (1-3 Hz) (Lakatos et al., 2008)], become entrained to the sensory inputs. In this situation, the high excitability phases of these oscillations tend to coincide with the temporal structures of the stimuli, consequently amplifying neural input responses (Lakatos et al., 2008; Schroeder and Lakatos, 2009), as such modulating signal-tonoise ratios. It is notable in this respect that we also observed a mental training-related increase in phase locking of lowfrequency oscillatory activity $(1-3 \mathrm{~Hz})$ to both attended and unattended stimuli (Fig. 4). Although this effect was not maximal at the intrinsic oscillatory mode of the stimuli stream $(0.85-1.3 \mathrm{~Hz})$, it may include an amplified entrainment of neuronal oscillations to sensory input rhythms in the delta frequency band. The assumption about the rhythmic mode attention during FA should be investigated further, in particular by looking at the influence of sensory input rhythms on the phase of the delta frequency band oscillations and by contrasting FA meditation with open monitoring meditation. OM meditation cultivates a monitoring state that does not focus or hold attention from moment to moment on an explicit object (Lutz et al., 2008a; Slagter et al., 2009). We would therefore predict that attention will operate less in a rhythmic mode during this state than during FA meditation.

The observed increase in phase consistency to deviant tones in general cannot simply be explained as a general mental trainingrelated increase in arousal level for two reasons. First, as noted above, we observed a reduction in cortical engagement, as reflected by a mental training-related decrease in ERD, and effort to do a task is typically positively linked to some increase in arousal (Critchley et al., 2003). Second, both groups showed an increase in global alpha power over time, and increases in alpha are generally associated with decreased arousal (Niedermeyer, 1999). To summarize, FA meditation may have reduced cortical signal-tonoise (e.g., PLF) in attention-related networks or may have led attention to operate more steadily into a rhythmic mode, thereby increasing signals to task-relevant stimuli. Future studies are necessary to distinguish between these two accounts.

\section{Limitations of the study and future directions}

One important limitation of our study is that subjects selfselected to participate in the 3 month retreat. Although the controls were also interested in learning to meditate, it is possible that the two groups differed in some variable before the study. However, no differences in performance on the sustained attention task were observed between the two groups at time 1. Future studies should randomly assign participants to the retreat group or the control group or should use a randomized wait-list control group design.

\section{Summary/conclusions}

To summarize, the current findings suggest that three months of intensive training in FA meditation (1) enhances attentional stability, (2) reduces task effort, and (3) amplifies the phase consistency of the brain responses to task-related sensory inputs. We propose that these mental training-related effects might be pro- 
duced by a reduction in cortical noise and/or by an enhancement of the rhythmic mode of attention. Future studies will have to test these ideas and examine to what extent purely mental training of attentional skills can also impact other mental domains, which depend on attention regulation, such as emotion regulation and self-regulation.

\section{References}

Aston-Jones G, Rajkowski J, Cohen J (1999) Role of locus coeruleus in attention and behavioral flexibility. Biol Psychiatry 46:1309-1320.

Brefczynski-Lewis JA, Lutz A, Schaefer HS, Levinson DB, Davidson RJ (2007) Neural correlates of attentional expertise in long-term meditation practitioners. Proc Natl Acad Sci U S A 104:11483-11488.

Cahn BR, Polich J (2009) Meditation (vipassana) and the P3a event-related brain potential. Int J Psychophysiol 72:51-60.

Castellanos FX, Sonuga-Barke EJ, Milham MP, Tannock R (2006) Characterizing cognition in ADHD: beyond executive dysfunction. Trends Cogn Sci 10:117-123.

Christoff K, Gordon AM, Smallwood J, Smith R, Schooler JW (2009) Experience sampling during fMRI reveals default network and executive system contributions to mind wandering. Proc Natl Acad Sci U S A 106:8719-8724.

Critchley HD, Mathias CJ, Josephs O, O’Doherty J, Zanini S, Dewar BK, Cipolotti L, Shallice T, Dolan RJ (2003) Human cingulate cortex and autonomic control: converging neuroimaging and clinical evidence. Brain 126:2139-2152.

Delorme A, Makeig S (2004) EEGLAB: an open source toolbox for analysis of single-trial EEG dynamics including independent component analysis. J Neurosci Methods 134:9-21.

Delorme A, Sejnowski T, Makeig S (2007) Enhanced detection of artifacts in EEG data using higher-order statistics and independent component analysis. Neuroimage 34:1443-1449.

Doyle LM, Yarrow K, Brown P (2005) Lateralization of event-related beta desynchronization in the EEG during pre-cued reaction time tasks. Clin Neurophysiol 116:1879-1888.

Gunaratana H (2002) Mindfulness in plain English. Somerville, MA: Wisdom Publications.

Gyatso, Tenzin (the XIV Dalai Lama), Jinpa GT (1995) The world of Tibetan Buddhism: an overview of its philosophy and practice. Somerville, MA: Wisdom Publications.

Hanslmayr S, Pastötter B, Bäuml KH, Gruber S, Wimber M, Klimesch W (2008) The electrophysiological dynamics of interference during the stroop task. J Cogn Neurosci 20:215-225.

Hillyard SA, Hink RF, Schwent VL, Picton TW (1973) Electrical signs of selective attention in the human brain. Science 182:177-180.

Jensen O, Lisman JE (1996) Theta/gamma networks with slow NMDA channels learn sequences and encode episodic memory: role of NMDA channels in recall. Learn Mem 3:264-278.

Jha AP, Krompinger J, Baime MJ (2007) Mindfulness training modifies subsystems of attention. Cogn Affect Behav Neurosci 7:109-119.

Jones MR, Boltz M (1989) Dynamic attending and responses to time. Psychol Rev 96:459-491.

Jones MR, Johnston HM, Puente J (2006) Effects of auditory pattern structure on anticipatory and reactive attending. Cogn Psychol 53:59-96.

Kahana MJ, Seelig D, Madsen JR (2001) Theta returns. Curr Opin Neurobiol 11:739-744.

Kelly AM, Uddin LQ, Biswal BB, Castellanos FX, Milham MP (2008) Competition between functional brain networks mediates behavioral variability. Neuroimage 39:527-537.

Kuntsi J, Stevenson J (2001) Psychological mechanisms in hyperactivity. II. the role of genetic factors. J Child Psychol Psychiatry 42:211-219.

Lakatos P, Karmos G, Mehta AD, Ulbert I, Schroeder CE (2008) Entrain- ment of neuronal oscillations as a mechanism of attentional selection. Science 320:110-113.

Lindholm E, Koriath JJ (1985) Analysis of multiple event related potential components in a tone discrimination task. Int J Psychophysiol 3:121-129.

Loh M, Rolls ET, Deco G (2007) A dynamical systems hypothesis of schizophrenia. PLoS Comput Biol 3:e228.

Lutz A, Brefczynski-Lewis J, Johnstone T, Davidson RJ (2008a) Regulation of the neural circuitry of emotion by compassion meditation: effects of meditative expertise. PLoS One 3:e1897.

Lutz A, Slagter HA, Dunne JD, Davidson RJ (2008b) Attention regulation and monitoring in meditation. Trends Cogn Sci 12:163-169.

Manassis K, Tannock R, Masellis M (1996) Cognitive differences between anxious, normal, and ADHD children on a dichotic listening task. Anxiety 2:279-285.

Nichols TE, Holmes AP (2002) Nonparametric permutation tests for functional neuroimaging: a primer with examples. Hum Brain Mapp 15:1-25.

Niedermeyer E (1999) The normal EEG of the waking adult. In: Electroencephalography: basic principles, clinical application, and related fields (Niedermeyer E, Lopes Da Silva F, eds). Baltimore: Lippincott Williams and Wilkins.

Palva JM, Palva S, Kaila K (2005) Phase synchrony among neuronal oscillations in the human cortex. J Neurosci 25:3962-3972.

Pfurtscheller G, Lopes da Silva FH (1999) Event-related EEG/MEG synchronization and desynchronization: basic principles. Clin Neurophysiol 110:1842-1857.

Polich J (2007) Updating P300: an integrative theory of P3a and P3b. Clin Neurophysiol 118:2128-2148.

Polich J, Criado JR (2006) Neuropsychology and neuropharmacology of P3a and P3b. Int J Psychophysiol 60:172-185.

Rolls ET, Loh M, Deco G, Winterer G (2008) Computational models of schizophrenia and dopamine modulation in the prefrontal cortex. Nat Rev Neurosci 9:696-709.

Romero SG, McFarland DJ, Faust R, Farrell L, Cacace AT (2008) Electrophysiological markers of skill-related neuroplasticity. Biol Psychol 78:221-230.

Schroeder CE, Lakatos P (2009) Low-frequency neuronal oscillations as instruments of sensory selection. Trends Neurosci 32:9-18.

Sinkkonen J, Tiitinen H, Näätänen R (1995) Gabor filters: an informative way for analysing event-related brain activity. J Neurosci Methods 56:99-104.

Slagter HA, Lutz A, Greischar LL, Francis AD, Nieuwenhuis S, Davis JM, Davidson RJ (2007) Mental training affects distribution of limited brain resources. PLoS Biol 5:e138.

Slagter HA, Lutz A, Greischar LL, Nieuwenhuis S, Davidson RJ (2009) Theta phase synchrony and conscious target perception: impact of intensive mental training. J Cogn Neurosci 21:1536-1549.

Swets JA, Green DM, Getty DJ, Swets JB (1978) Signal detection and identification at successive stages of observation. Percept Psychophys 23:275-289.

Tang YY, Ma Y, Wang J, Fan Y, Feng S, Lu Q, Yu Q, Sui D, Rothbart MK, Fan M, Posner MI (2007) Short-term meditation training improves attention and self-regulation. Proc Natl Acad Sci U S A 104:17152-17156.

Tiitinen H, Sinkkonen J, Reinikainen K, Alho K, Lavikainen J, Näätänen R (1993) Selective attention enhances the auditory 40-hz transient response in humans. Nature 364:59-60.

Weissman DH, Roberts KC, Visscher KM, Woldorff MG (2006) The neural bases of momentary lapses in attention. Nat Neurosci 9:971-978.

Winterer G, Ziller M, Dorn H, Frick K, Mulert C, Wuebben Y, Herrmann WM, Coppola R (2000) Schizophrenia: reduced signal-to-noise ratio and impaired phase-locking during information processing. Clin Neurophysiol 111:837-849. 